\title{
Geometric frustration in the mixed layer pnictide oxides
}

\author{
Matthew Enjalran \\ Department of Physics, University of California, Davis, CA 95616 \\ and Materials Research Institute, Lawrence Livermore National Laboratory, \\ University of California, Livermore, CA 94550 \\ Richard T. Scalettar \\ Department of Physics, University of California, Davis, CA 95616 \\ Susan M. Kauzlarich \\ Department of Chemistry, University of California, Davis, CA 95616
}

(November 21, 2018)

We present results from a Monte Carlo investigation of a simple bilayer model with geometrically frustrated interactions similar to those found in the mixed layer pnictide oxides $\left(\mathrm{Sr}_{2} \mathrm{Mn}_{3} \mathrm{Pn}_{2} \mathrm{O}_{2}, \mathrm{Pn}=\mathrm{As}, \mathrm{Sb}\right)$. Our model is composed of two inequivalent square lattices with nearest neighbor intraand interlayer interactions. We find a ground state composed of two independent Néel ordered layers when the interlayer exchange is an order of magnitude weaker than the intralayer exchange, as suggested by experiment. We observe this result independent of the number of layers in our model. We find evidence for local orthogonal order between the layers, but it occurs in regions of parameter space that are not experimentally realized. We conclude that frustration caused by nearest neighbor interactions in the mixed layer pnictide oxides is not sufficient to explain the long-range orthogonal order that is observed experimentally, and that it is likely that other terms (e.g., local anisotropies) in the Hamiltonian are required to explain the magnetic behavior.

\section{INTRODUCTION}

Clean systems of interacting moments have been studied extensively by analytic and numerical techniques. Although simplified models like Ising, Heisenberg, and Hubbard retain only the most fundamental interactions observed in real materials, they remain tractable to current theoretical techniques, and the study of their ordered phases in various regions of parameter space has contributed enormously to our understanding of magnetic phenomena and the physics of correlated systems. [1] However, real materials are never clean. There is often frustration due to competing interactions and disorder in the interaction strengths.

Competing interactions that cause magnetic frustration can have many origins, lattice geometry, magnetic and non-magnetic impurities. In three dimensions, helical magnetic order has been observed when geometric frustration is accompanied by anisotropy. [2,3] Spin glasses phases are observed when frustration is accompanied by random disorder. [1 6] It has also been suggested that some non-collinear spin ordered structures belong to a new chiral universality class. [7] The systems we study are essentially two dimensional and contain no anisotropic terms or disorder. Frustration is caused by the lattice geometry. Our primary focus is the orthogonal magnetic structure observed in the mixed layer pnictide oxides. [8]

The pnictide oxides of type $A_{2} M n_{3} \mathrm{Pn}_{2} \mathrm{O}_{2}(\mathrm{~A}=$ $B a, S r ; P n=A s, S b)$ are layered antiferromagnets that contain two distinct square planes of manganese atoms arranged in a lattice of space group symmetry $I 4 / \mathrm{mmm}$. In one layer, manganese is bonded to oxygen in a planar $\mathrm{CuO}_{2}$ arrangement, $\mathrm{MnO}_{2}^{2-}$. In a second layer, it is bonded to a pnictogen in a tetrahedral structure, $M n P n_{2}^{2-}$, where pnictogen atoms project alternately above and below the plane defined by the manganese atoms. From here on we denote the two layers as $\mathrm{Mn}(1)$ for $\mathrm{MnO}_{2}^{2-}$ and $\mathrm{Mn}(2)$ for $\mathrm{MnPn}_{2}^{2-}$. The $\mathrm{Mn}$ atoms from the two planes are arranged so that a site in the $\mathrm{Mn}(1)$ layer sits directly above and below the center of a square plaquette of $\mathrm{Mn}$ atoms in the $\mathrm{Mn}(2)$ layer. The manganese carry a spin $S=5 / 2$. Frustration can enter through nearest neighbor interlayer coupling. A more detailed investigation of these systems has been reported elsewhere. [9]

\section{MODEL}

In all the pnictide oxides except $\mathrm{Sr}_{2} \mathrm{Mn}_{3} \mathrm{As}_{2} \mathrm{O}_{2}$ there is long range order in the planes that eventually gives rise to weak $3 \mathrm{D}$ order. In the compound $\mathrm{Sr}_{2} \mathrm{Mn}_{3} \mathrm{As}_{2} \mathrm{O}_{2}$, there is only short range order in the $\mathrm{Mn}(1)$ planes. The ability of ordered planes to drive c-axis order has been investigate before in the case of layered antiferromagnets. 10 12 In $\mathrm{Sr}_{2} \mathrm{Mn}_{3} \mathrm{Sb}_{2} \mathrm{O}_{2}$, magnetic order in the $\mathrm{Mn}(1)$ layers is established along the a-axis of the magnetic unit cell, while in the $\operatorname{Mn}(2)$ layers the magnetization is along the c-axis. Hence there is an orthogonal alignment between neighboring layers. Such an ordered state is not without precedent. [13, 14, However, the different temperatures at which the layers order $\left(T_{M n 2} \approx 300 K\right.$ and $\left.T_{M n 1} \approx 65 K\right)$ and the symmetry of 
the frustrated interlayer interactions which leads to cancellation suggest a system of two independent Néel ordered layers. Previous work has shown that this is not always the case, as thermal or quantum fluctuations (in frustrated systems) can lift the degeneracy of the system to select a single state. 15, 16

To study the effect of frustration on the ground state magnetic order of the pnictide oxides, we developed a simple model of classical Heisenberg spins with nearest neighbor intra- and interlayer interactions. The basic structural unit is a set of two layers, one each of type $\operatorname{Mn}(1)$ and $M n 2$ (see Fig. 1). The $\operatorname{Mn}(2)$ layer has a lattice constant $a=1$ and contains $n^{2}$ sites. The $\operatorname{Mn}(1)$ layer is larger by a factor $\sqrt{2}$ and is rotated by $\pi / 4$ with respect to the lattice directions of the other layer. The $\operatorname{Mn}(1)$ layer contains $n^{2} / 2+n+1$ spins. Note that the interlayer coordination is not the same for spins on the two layers. A spin on the $\mathrm{Mn}(1)$ plane is coupled to four spins on the $\operatorname{Mn}(2)$ plane, and each spin in the $\operatorname{Mn}(2)$ plane is coupled to only two spins on the $\mathrm{Mn}(1)$ plane.

The Hamiltonian for our bilayer model is written as

$$
\begin{aligned}
H & =J_{1} \sum_{i, \vec{\delta}_{1}} \vec{S}_{i}^{(1)} \cdot \vec{S}_{i+\vec{\delta}_{1}}^{(1)}+J_{2} \sum_{i, \vec{\delta}_{2}} \vec{S}_{i}^{(2)} \cdot \vec{S}_{i+\vec{\delta}_{2}}^{(2)} \\
& +J_{\perp} \sum_{i, \vec{\delta}_{\perp}} \vec{S}_{i}^{(\alpha)} \cdot \vec{S}_{i+\vec{\delta}_{\perp}}^{(\beta)} \cdot
\end{aligned}
$$

The constants $J_{1}, J_{2}$, and $J_{\perp}$ represent the $\operatorname{Mn}(1)$ and $\operatorname{Mn}(2)$ intralayer couplings and the interlayer coupling, respectively. The summations of $\vec{\delta}_{\mu}$ are over nearest neighbors to site $i$. For classical spins, one has $|\vec{S}|=$ $\left(S_{x}^{2}+S_{y}^{2}+S_{z}^{2}\right)^{1 / 2}=1$. The relatively large spin-5/2 of the $\mathrm{Mn}$ atoms in the pnictide oxides makes this a reasonable approximation.

We studied the equilibrium physics of our model by a single spin flip Monte Carlo algorithm. We have addressed concerns about proper sampling of phase space by performing simulations with random and ordered initial configurations. We have also considered the effects of the boundary on our finite simulations by employing a few different boundary conditions: open, periodic, and periodic with an effective field on the $\mathrm{Mn}(1)$ edge sites. In all cases considered, we found no qualitative difference in our results due to the initial configuration or the conditions imposed at the boundary.

To determine the relative orientation between neighboring spins, either within the same layer or in different layers, we measured a collinear

$$
C_{\|}^{\alpha, \beta}=\left\langle\frac{1}{z N_{\alpha}} \sum_{i} \sum_{\vec{\delta}}\left(\vec{S}_{i}^{\alpha} \cdot \vec{S}_{i+\vec{\delta}}^{\beta}\right)^{2}\right\rangle,
$$

and a perpendicular

$$
C_{\perp}^{\alpha, \beta}=\left\langle\frac{1}{z N_{\alpha}} \sum_{i} \sum_{\vec{\delta}}\left(\vec{S}_{i}^{\alpha} \times \vec{S}_{i+\vec{\delta}}^{\beta}\right)^{2}\right\rangle
$$

spin-spin correlation function. Here summations are performed over all nearest neighbors $\vec{\delta}$ of site i and then over all sites in the lattice; $z$ is the coordination number and $N_{\alpha}$ is the number of sites in layer $\alpha$. Intralayer correlations are denoted by $\alpha=\beta$ and interlayer correlations are represented by $\alpha \neq \beta$. We stress that $C_{\|}$and $C_{\perp}$ measure local correlations. For classical Heisenberg spins, these correlations take on the simple forms $C_{\|}=\left\langle\cos ^{2} \theta\right\rangle$ and $C_{\perp}=\left\langle\sin ^{2} \theta\right\rangle$. In the high temperature, paramagnetic, limit, the values $C_{\|}=1 / 3$ and $C_{\perp}=2 / 3$ are obtained. We also measured the magnetization and staggered magnetization of each layer.

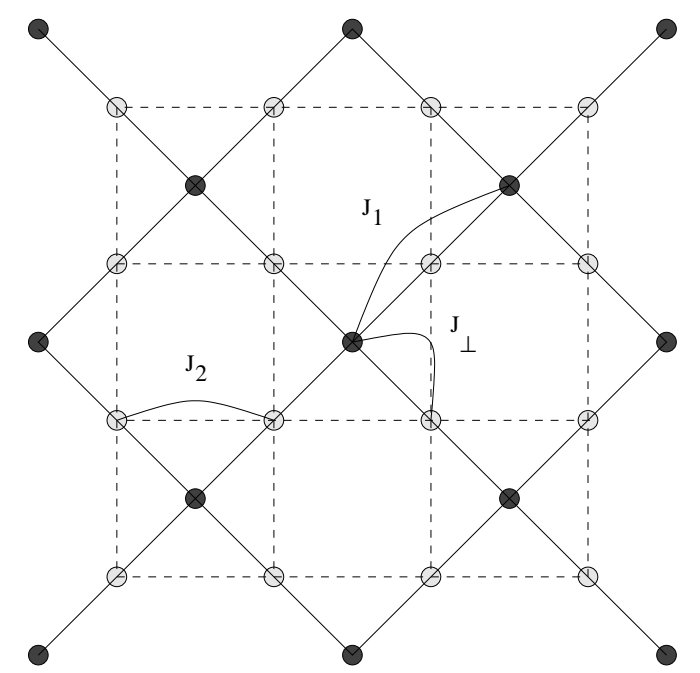

FIG. 1. A 2D projection of the two distinct layers of the pnictide oxide $\mathrm{Sr}_{2} \mathrm{Mn}_{3} \mathrm{Sb}_{2} \mathrm{O}_{2}$. Sites in the $\mathrm{Mn}(1)$ layer are represented by dark circles while sites in the $\mathrm{Mn}(2)$ layer are represented by light circles. The intralayer couplings are shown as $J_{1}$ and $J_{2}$, and the interlayer interaction is indicated by $J_{\perp}$.

\section{RESULTS AND DISCUSSION}

From the experimental data, representative couplings would set the $\operatorname{Mn}(2)$ intralayer exchange to be stronger than the $\mathrm{Mn}(1)$ intralayer exchange, with the interlayer interaction weaker by at least an order of magnitude. Therefore, experimentally motivated couplings in our model were set to $J_{2}=2.0, J_{1}=1.0$ and $J_{\perp}=0.1$. For the results presented herein, systems with 1600 spins per $\mathrm{Mn}(1)$ layer and 841 spins per $\mathrm{Mn}(1)$ were equilibrated for 15,000 to 25,000 sweeps followed by 15, 000 measurement sweeps with $10-25$ sweeps between measurements. One Monte Carlo sweep denotes an update of all spins on the lattice.

Our results for a bilayer model indicate that, as a function of temperature, the moments within each layer began to order when the temperature dropped below the respective energy scale, e.g., $T=J_{1}$ for $\mathrm{Mn}(1)$ moments and $T=J_{2}$ for $\operatorname{Mn}(2)$ moments. However, the eventual ground state was a system of two Néel ordered layers 
with an arbitrary orientation between the magnetization directions. In a simulation with four layers (i.e., a layered sequence $M n 1-M n 2-M n 1-M n 2)$ and periodic boundary conditions along the c-axis, we observed the same qualitative behavior as a function of temperature (see Fig. 2). We emphasize that the interlayer spin-spin correlation function, $C_{\|}^{1,2}$, remained at the paramagnetic limit down to low temperatures, $T \lesssim J_{\perp}$.

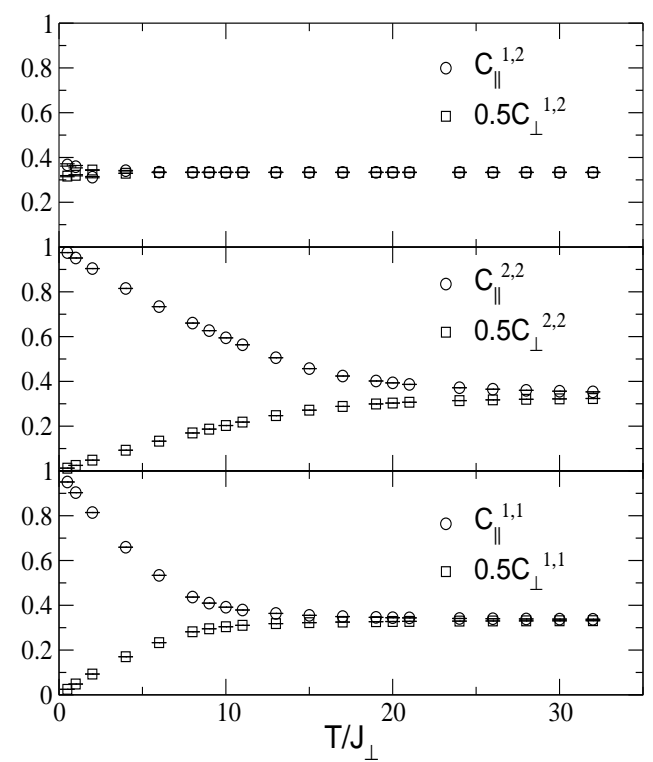

FIG. 2. Temperature dependence of the local intra- and interlayer spin-spin correlations in the four layer model with periodic boundary conditions and $J_{1}=1.0, J_{2}=2.0, J_{\perp}=0.1$. A parallel alignment is favored for intralayer spins when the temperature drops below the respective intralayer coupling; however, the interlayer correlations remain at the high temperature limit of $1 / 3$ even for $T \lesssim J_{\perp}$.

We also studied the effect of the strength of frustration on the magnetic ground state. To do this, we fixed the temperature and swept in values of $J_{\perp}$. For a bilayer model with $J_{\perp}<0.25$, we observed two Néel ordered layers with a paramagnetic interlayer orientation, i.e., $C_{\|}^{1,2} \approx 1 / 3$ or independent layers. As $J_{\perp}$ was increased, the two Néel ordered layers moved towards a collinear state. We observed this behavior independent of the initial configuration (see Fig. 3).

An explanation of these results can be understood by considering the physics of two simpler models, a zigzag lattice and a diagonal lattice, (refer to Ref. [9]). In the zigzag lattice the frustration is along one crystalline direction; this geometry is similar to the $\operatorname{Mn}(2)$ to $\operatorname{Mn}(1)$ interlayer interaction. In the limit of weak $J_{\perp}$, a uniformly canted state is established in the zigzag lattice with orthogonal order obtained in the limit $J_{\perp} \rightarrow 0$. In the diagonal lattice, one has the equivalent of the $J_{1}-J_{2}$ model. This interlayer geometry is analogous to the coupling of $\mathrm{Mn}(1)$ sites to $\mathrm{Mn}(2)$ sites. In the limit of weak $J_{\perp}$, the diagonal lattice is composed of two Néel ordered layers with a collinear alignment. Hence, at weak frustration, it is the competition between these two tendencies in the experimental model that leads to a paramagnetic orientation with large fluctuations. In the case of strong frustration, $J_{\perp}>0.5$, the zigzag and diagonal geometries both select a collinear interlayer arrangement.

An orthogonal state for our bilayer model can be found but this phase occurs at couplings strengths that are not supported by experiments (refer to Ref. [9]). In the case where $J_{2}=0$, the resultant model is a network of intersecting zigzag chains. By setting $J_{\perp}=1$ and sweeping in $J_{1}$ a transition to a uniformly canted state was observed in the limit of large $J_{1}$. For a model with $J_{2}=J_{\perp}=1.0$, we observed a Néel ordered $\operatorname{Mn}(2)$ layer and a paramagnetic $\operatorname{Mn}(1)$ layer, but with $\mathrm{Mn}(1)$ spins orthogonal to the local $\mathrm{Mn}(2)$ environment, for $J_{1} \leq 0.25$. At $J_{1}>0.25$ the system tended toward a state with collinear alignment.

We conclude that frustration caused by nearest neighbor interactions, both intra- and interlayer, in the mixed layer pnictide oxides is not sufficient to explain the long range orthogonal order that is observed experimentally. However, recent work on mixed metal pnictide oxides does indicate that the two distinct layers do not behave independently. [17] In these systems, it is likely that other terms in the Hamiltonian, e.g., ion anisotropies arising from spin-orbit effects, are required to explain the magnetic behavior.

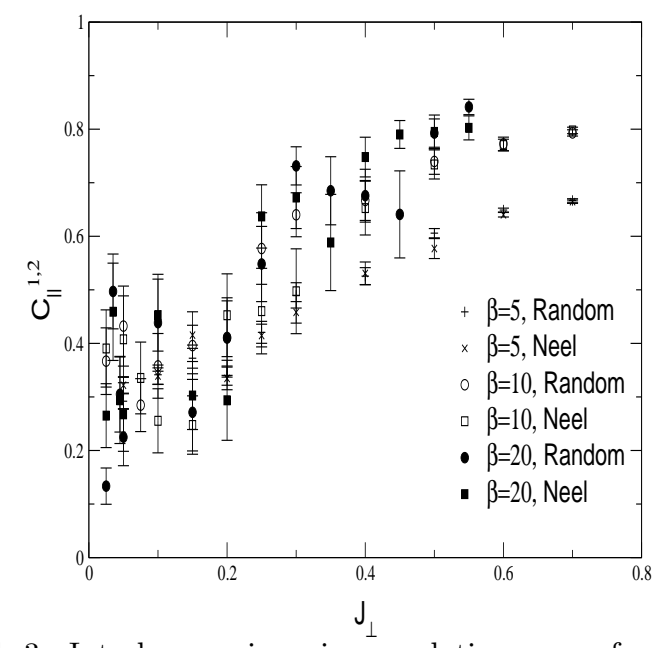

FIG. 3. Interlayer spin-spin correlations as a function of $J_{\perp}$ with $J_{1}=1.0$ and $J_{2}=2.0$. The results are for a bilayer model with the initial configuration of the layers being either random or Néel ordered.

We acknowledge the generous support of CampusLaboratory Collaboration of the University of California, the Materials Research Institute at Lawrence Livermore National Laboratory. We thank Rajiv Singh and Tadashi Ozawa at the University of California, Davis for many useful discussions. We also thank D.P. Landau at the Center for Simulational Physics at the University of Georgia, Athens for helpful suggestions. Work at Lawrence Livermore National Laboratory performed un- 
der the auspices of the U.S. Department of Energy under contract number W-7405-ENG-48.

[1] D.C. Mattis, The Theory of Magnetism II, Thermodynamics and Statistical Mechanics, edited by P. Fulde, (Springer-Verlag, Berlin, 1985); A. Auerbach, Interacting Electrons and Quantum Magnetism, edited by J.L. Birman, H. Faissner, and J.W. Lynn, (SpringerVerlag, New York, 1994); F. Gebhard, The Mott MetalInsulator Transition, edited by G. Höhler, (SpringerVerlag, Berlin, 1997).

[2] M.L. Plumer, A. Caillé, and H.T. Diep, in Magnetic Systems with Competing Interactions (Frustrated Spin Systems), edited by H.T. Diep, (World Scientific, 1994).

[3] H.T. Diep, Europhys. Lett. 7, 725 (1988); H.T. Diep, Phys. Rev. B 39, 397 (1989).

[4] K. Binder and A.P. Young, Rev. Mod. Phys. 58, 801 (1986).

[5] Spin Glasses and Random Fields, edited by A.P. Young, (World Scientific, River Edge, N.J., 1998).

[6] M.J.P. Gingras, in Magnetic Systems with Competing Interactions (Frustrated Spin Systems), edited by H.T. Diep, (World Scientific, 1994).

[7] H. Kawamura, J. Phys. Soc. Jpn. 54, 3220 (1985); H. Kawamura, Phys. Rev. B 38, 4916 (1988).

[8] S.L. Brock, N.P. Raju, J.E. Greedan, and S.M. Kauzlarich, J. Alloys Comp. 237, 9 (1996); S.L. Brock and S.M. Kauzlarich, J. Alloys Comp. 241, 82 (1996); S.L. Brock and S.M. Kauzlarich, Chemtech 25, 18 (1995).

[9] M. Enjalran, R.T. Scalettar, and S. M. Kauzlarich, Phys. Rev. B 61, 14570 (2000).

[10] M.E. Lines, Phys. Rev. 164, 736 (1967); M.E. Lines, J. Phys. Chem. Solids 31, 101 (1970);

[11] R.J. Birgeneau, H.J. Guggenheim, and G. Shirane, Phys. Rev. Lett. 22, 720 (1969).

[12] R.J. Birgeneau, H.J. Guggenheim, and G. Shirane, Phys. Rev. B 1, 2211 (1970); R.J. Birgeneau, H.J. Guggenheim, and G. Shirane, Phys. Rev. B 8, 304 (1973).

[13] J. Leciejewicz, S. Siek, and A. Szytula, J. Magn. Mater. 40, 265 (1984).

[14] C.C. Torardi, W.M. Reiff, K. Lázár, J.H. Zhang, and D.E. Cox, J. Solid State Chem. 66, 105 (1987).

[15] C. Henley, Phys. Rev. Lett. 62, 2056 (1989).

[16] A. Moreo, E. Dagotto, T. Jolicoeur, and J. Riera, Phys. Rev. B 42, 6283 (1990).

[17] T. Ozawa, M.M. Olmstead, S.L. Brock, S.M. Kauzlarich, and D.M. Young, Chem. Mater. 10, 392 (1998); T. Ozawa, S.M. Kauzlarich, M. Bieringer, C.R. Wiebe, J.E. Greedan, and J.S. Gardner, submitted to Chem. Mater. 